J. Korean Math. Soc. 50 (2013), No. 3, pp. 591-605

http://dx.doi.org/10.4134/JKMS.2013.50.3.591

\title{
DERIVATIONS OF THE ODD CONTACT LIE ALGEBRAS IN PRIME CHARACTERISTIC
}

\author{
Yan CaO, Xiumei Sun, And JiXia Yuan
}

\begin{abstract}
The underlying field is of characteristic $p>2$. In this paper, we first use the method of computing the homogeneous derivations to determine the first cohomology of the so-called odd contact Lie algebra with coefficients in the even part of the generalized Witt Lie superalgebra. In particular, we give a generating set for the Lie algebra under consideration. Finally, as an application, the derivation algebra and outer derivation algebra of the Lie algebra are completely determined.
\end{abstract}

\section{Introduction}

In this paper, we consider a family of non-simple modular Lie algebras, called the odd contact Lie algebras, which are actually the even parts of the odd contact simple Lie superalgebras that are also closely related to the contact simple Lie algebras. The main purpose is to compute the first cohomology of the odd contact Lie algebras with coefficients in their adjoint representations. In other words, we determine the derivation algebras and the outer derivation algebras.

As is well-known, the theory of modular Lie algebras has undergone a remarkable evolution. In the super-case, the theory of modular Lie superalgebras has also obtained many interesting results in the past decade. For example, one can find work on the classification of classical modular Lie superalgebras $[1,2]$ and on the structures and representations of modular Lie superalgebras of Cartan type $[6,7,9,16,17,18]$. Recently, one can also find work on the representations of the classical modular Lie superalgebras (see, for example, $[14,15])$. For the simple modular Lie algebras and simple modular Lie superalgebras of Cartan type, the (super)derivation algebras have been sufficiently studied (for example, see $[3,4,8,9,10,11,12,13]$ ). In [4, 11], the superderivation algebras of Lie superalgebras of Cartan-type $H, W, S, K, H O$ and $K O$ were determined. For the derivations of the even parts of Lie superalgebras

Received August 25, 2012.

2010 Mathematics Subject Classification. 17B50, 17B40.

Key words and phrases. Lie superalgebra, derivation, first cohomology.

The second author was supported by the NSF of Heilongjiang Province, China (A200903) and the NSF of China (10871057). 
of Cartan type $W, S$ and $H O$, we refer the reader to $[8,10]$. Our work is essentially motivated by the work on modular Lie algebras of Cartan type (see $[3,12,13])$.

This paper is organized as follows. In Section 1, we recall some notions and basic facts and state the main results (Theorems 1.1 and 1.2). The rest of the paper covers the proofs of Theorems 1.1 and 1.2. In Section 2, we give a generating set of the odd contact Lie algebra and recall the so-called $\mathbb{Z}$ grading structures, which will be frequently used, and establish some technical lemmas pertaining to transitivity for computing derivations. In Section 3, we determine the derivation space from the odd contact Lie algebra into the even part of generalized Witt superalgebra as module. As an application, we finally complete the characterization of the derivation algebra and the outer derivation algebra.

\section{Notation and main results}

Throughout the present paper, $\mathbb{F}$ is a field of prime characteristic $p>2$, and $\mathbb{N}$ and $\mathbb{N}_{0}$ are the sets of positive integers and non-negative integers, respectively. Let $\mathbb{Z}_{2}:=\{\overline{0}, \overline{1}\}$ be the additive group of order 2 . We first give a description of the graded Lie superalgebras of Cartan type. For an $n$-tuple $\alpha:=\left(\alpha_{1}, \ldots, \alpha_{n}\right) \in \mathbb{N}_{0}^{n}$, put $|\alpha|:=\sum_{i=1}^{n} \alpha_{i}$. Fix $\underline{t}:=\left(t_{1}, t_{2}, \ldots, t_{n}\right) \in \mathbb{N}^{n}$ and write $\pi:=\left(\pi_{1}, \pi_{2}, \ldots, \pi_{n}\right)$, where $\pi_{i}:=p^{t_{i}}-1, i \in \overline{1, n}$. Let $\mathbb{A}(n, \underline{t}):=\{\alpha \in$ $\left.\mathbb{N}_{0}^{n} \mid \alpha_{i} \leq \pi_{i}, i \in \overline{1, n}\right\}$. Denote by $\mathcal{O}(n ; \underline{t})$ the divided power algebra over $\mathbb{F}$ with $\mathbb{F}$-basis $\left\{x^{(\alpha)} \mid \alpha \in \mathbb{A}(n, \underline{t})\right\}$. For $\varepsilon_{i}:=\left(\delta_{i 1}, \delta_{i 2}, \ldots, \delta_{i n}\right)$, we write $x_{i}$ for $x^{\left(\varepsilon_{i}\right)}$, $i \in \overline{1, n}$. Let $\Lambda(n+1)$ be the exterior superalgebra over $\mathbb{F}$ in $n+1$ variables $x_{n+1}, x_{n+2}, \ldots, x_{2 n+1}$. The tensor product $\mathcal{O}(n, n+1 ; \underline{t}):=\mathcal{O}(n ; \underline{t}) \otimes_{\mathbb{F}} \Lambda(n+1)$ is an associative superalgebra in the natural way, which is called a divided power superalgebra. For $g \in \mathcal{O}(n ; \underline{t})$ and $f \in \Lambda(n+1)$, we simply write $f g$ to mean $f \otimes g$. Recall the multiplication in $\mathcal{O}(n, n+1 ; \underline{t})$ :

$$
\begin{aligned}
& x^{(\alpha)} x^{(\beta)}=\left(\begin{array}{c}
\alpha+\beta \\
\alpha
\end{array}\right) x^{(\alpha+\beta)} \quad \text { for } \alpha, \beta \in \mathbb{A}(n, \underline{t}), \\
& x_{i} x_{j}=-x_{j} x_{i} \text { for } i, j \in \overline{n+1,2 n+1}, \\
& x^{(\alpha)} x_{j}=x_{j} x^{(\alpha)} \quad \text { for } \alpha \in \mathbb{A}(n, \underline{t}), j \in \overline{n+1,2 n+1},
\end{aligned}
$$

where

$$
\left(\begin{array}{c}
\alpha+\beta \\
\alpha
\end{array}\right)=\prod_{i=1}^{n}\left(\begin{array}{c}
\alpha_{i}+\beta_{i} \\
\alpha_{i}
\end{array}\right) .
$$

Let $\mathbb{B}_{k}:=\left\{\left\langle i_{1}, i_{2}, \ldots, i_{k}\right\rangle \mid n+1 \leq i_{1}<i_{2}<\cdots<i_{k} \leq 2 n+1\right\}$ and

$$
\mathbb{B}:=\mathbb{B}(n+1):=\cup_{k=0}^{n+1} \mathbb{B}_{k},
$$

where $\mathbb{B}_{0}:=\emptyset$. Set $\mathbb{B}^{0}:=\{u \in \mathbb{B}|| u \mid$ is even $\}$ and $\mathbb{B}^{1}:=\{u \in \mathbb{B}|| u \mid$ is odd $\}$. For $u:=\left\langle i_{1}, i_{2}, \ldots, i_{k}\right\rangle \in \mathbb{B}_{k}$, put $|u|:=k,\{u\}:=\left\{i_{1}, i_{2}, \ldots, i_{k}\right\}$, and $x^{u}:=$ $x_{i 1} x_{i 2} \cdots x_{i k}$. We write $|\emptyset|:=0, x^{\emptyset}:=1$ and $\omega:=\langle n+1, \ldots, 2 n+1\rangle$. Note that $\left\{x^{(\alpha)} x^{u} \mid \alpha \in \mathbb{A}(n, \underline{t}), u \in \mathbb{B}(n+1)\right\}$ is a $\mathbb{Z}_{2}$-homogeneous $\mathbb{F}$-basis of 
$\mathcal{O}(n, n+1 ; \underline{t})$. Denote the $\mathbb{Z}_{2}$-degree of a $\mathbb{Z}_{2}$-homogeneous element $x$ by $\mathrm{p}(x)$. Let $D_{1}, D_{2}, \ldots, D_{2 n+1}$ be the linear transformations of $\mathcal{O}(n, n+1 ; \underline{t})$ such that $D_{i}\left(x^{(\alpha)} x^{u}\right)=x^{\left(\alpha-\varepsilon_{i}\right)} x^{u}$ if $i \in \overline{1, n}$ and $x^{(\alpha)} \frac{\partial x^{u}}{\partial x_{i}}$ if $i \in \overline{n+1,2 n+1}$. Then $D_{1}, D_{2}, \ldots, D_{2 n+1}$ are superderivations of the superalgebra $\mathcal{O}(n, n+1 ; \underline{t})$ and $\mathrm{p}\left(D_{i}\right)=\mu(i)$, where $\mu(i):=\overline{0}$ if $i \in \overline{1, n}$ and $\overline{1}$ if $i \in \overline{n+1,2 n+1}$. Recall the generalized Witt superalgebra

$$
W(n, n+1 ; \underline{t}):=\left\{\sum_{i=1}^{2 n+1} a_{i} D_{i} \mid a_{i} \in \mathcal{O}(n, n+1 ; \underline{t}), i \in \overline{1,2 n+1}\right\},
$$

which is a finite-dimensional simple Lie superalgebra (see [16]). The even part of $W(n, n+1 ; \underline{t})$ is denoted by $\mathfrak{W}(n, n+1 ; \underline{t})$. Note that $W(n, n+1 ; \underline{t})$ is a free $\mathcal{O}(n, n+1 ; \underline{t})$-module with a basis $\left\{D_{1}, D_{2}, \ldots, D_{2 n+1}\right\}$. Put $i^{\prime}:=i+n$ if $i \in \overline{1, n}$ and $i-n$ if $i \in \overline{n+1,2 n}$. Define

$$
\begin{aligned}
\mathrm{T}_{\mathrm{K}}: \mathcal{O}(n, n+1 ; \underline{t}) \longrightarrow W(n, n+1 ; \underline{t}) \\
\mathrm{T}_{\mathrm{K}}(a):=\sum_{i=1}^{2 n}\left((-1)^{\mu\left(i^{\prime}\right) \mathrm{p}(a)} D_{i^{\prime}} a+(-1)^{\mathrm{p}(a)}\left(D_{2 n+1} a\right) x_{i}\right) D_{i} \\
+\left(\sum_{i=1}^{2 n} x_{i}\left(D_{i} a\right)-2 a\right) D_{2 n+1} .
\end{aligned}
$$

Then $\mathrm{T}_{\mathrm{K}}$ is an odd linear mapping and the following formula holds for $a, b \in$ $\mathcal{O}(n, n+1 ; \underline{t})$ :

$$
\left[\mathrm{T}_{\mathrm{K}}(a), \mathrm{T}_{\mathrm{K}}(b)\right]=\mathrm{T}_{\mathrm{K}}\left(\mathrm{T}_{\mathrm{K}}(a)(b)-(-1)^{\mathrm{p}(a)} 2\left(D_{2 n+1} a\right) b\right) .
$$

It follows that the image of $\mathrm{T}_{\mathrm{K}}$,

$$
K O(n, n+1 ; \underline{t}):=\left\{\mathrm{T}_{\mathrm{K}}(a) \mid a \in \mathcal{O}(n, n+1 ; \underline{t})\right\},
$$

is a finite-dimensional simple subalgebra of $W(n, n+1 ; \underline{t})$, known as the odd contact Lie superalgebra (see [4]). The even part of this Lie superalgebra is called the odd contact Lie algebra, denoted by $\mathfrak{K}(n, n+1 ; \underline{t})$.

For simplicity, in the sequel, the parameter tuple $(n, n+1 ; \underline{t})$ will be omitted. For example, we usually write $\mathcal{O}, \mathfrak{W}$ and $\mathfrak{K}$ for $\mathcal{O}(n, n+1 ; \underline{t}), \mathfrak{W}(n, n+1 ; \underline{t})$ and $\mathfrak{K}(n, n+1 ; \underline{t})$, respectively. View $\mathfrak{W}$ as a $\mathfrak{K}$-module by means of the adjoint representation. The primary results of this paper are:

Theorem 1.1. The derivation space from $\mathfrak{K}$ into $\mathfrak{W}$ is

$$
\operatorname{Der}(\mathfrak{K}, \mathfrak{W})=\operatorname{ad}(\mathfrak{W}) \oplus \operatorname{span}_{\mathbb{F}}\left\{\left(\operatorname{ad} D_{i}\right)^{p^{k_{i}}} \mid i \in \overline{1, n}, 1 \leq k_{i}<t_{i}\right\} .
$$

In particular, the first cohomology space of $\mathfrak{K}$ with values in $\mathfrak{W}$ is of dimension $\sum_{i=1}^{n} t_{i}-n$.

Theorem 1.2. The derivation algebra of the odd contact Lie algebra is

$$
\operatorname{Der}(\mathfrak{K})=\operatorname{ad}(\mathfrak{K}) \oplus \operatorname{span}_{\mathbb{F}}\left\{\left(\operatorname{ad} D_{i}\right)^{p^{k_{i}}} \mid i \in \overline{1, n}, 1 \leq k_{i}<t_{i}\right\} .
$$


In particular, the outer derivation algebra is an abelian Lie algebra of dimension $\sum_{i=1}^{n} t_{i}-n$.

\section{Generating set and grading structures}

\subsection{Generating set}

A derivation of a Lie algebra $L$ is completely determined by its action on a generating set of $L$. Thereby we first give a generating set of the odd contact Lie algebra, which will be frequently used in this paper. Put

$$
\begin{aligned}
& \mathcal{M}:=\left\{\mathrm{T}_{\mathrm{K}}\left(x_{n+i}\right) \mid i \in \overline{1, n}\right\}, \\
& \mathcal{N}:=\left\{\mathrm{T}_{\mathrm{K}}\left(x^{\left(k \varepsilon_{i}\right)} x_{2 n+1}\right) \mid i \in \overline{1, n}, 0 \leq k \leq \pi_{i}\right\}, \\
& \mathcal{P}:=\left\{\mathrm{T}_{\mathrm{K}}\left(x_{n+l} x_{n+t} x_{2 n+1}\right) \mid l, t \in \overline{1, n}\right\} .
\end{aligned}
$$

Theorem 2.1. $\mathcal{M} \cup \mathcal{N} \cup \mathcal{P}$ is a generating set of $\mathfrak{K}$.

Proof. Denote by $\mathcal{L}$ the subalgebra of $\mathfrak{K}$ generated by $\mathcal{M} \cup \mathcal{N} \cup \mathcal{P}$. For $\mathrm{T}_{\mathrm{K}}(a)$, $\mathrm{T}_{\mathrm{K}}(b) \in \mathcal{L}$, if neither $a$ nor $b$ contains $x_{2 n+1}$, then the Lie bracket of $\mathrm{T}_{\mathrm{K}}(a)$ and $\mathrm{T}_{\mathrm{K}}(b)$ equals to that of $\mathrm{T}_{\mathrm{H}}(a)$ and $\mathrm{T}_{\mathrm{H}}(b)$ in the even part of the odd Hamiltonian superalgebra $H O(n, n ; \underline{t}$ ) (see [11]). By [8, Proposition 2.4], the even part of $H O(n, n ; \underline{t})$ has a generating set $A$ :

$$
\begin{aligned}
& \left\{\mathrm{T}_{\mathrm{H}}\left(x_{k} x_{l} x_{q}\right) \mid k, l, q \in \overline{n+1,2 n}\right\} \\
\cup & \left\{\mathrm{T}_{\mathrm{H}}\left(x^{\left(q_{i} \varepsilon_{i}\right)} x_{k}\right) \mid i \in \overline{1, n}, 0 \leq q_{i} \leq \pi_{i}, k \in \overline{n+1,2 n}\right\} .
\end{aligned}
$$

Since $\left[\mathrm{T}_{\mathrm{K}}\left(x^{\left(k_{j} \varepsilon_{j}\right)} x_{2 n+1}\right), \mathrm{T}_{\mathrm{K}}\left(x_{n+i}\right)\right]=\mathrm{T}_{\mathrm{K}}\left(x^{\left(k_{j} \varepsilon_{j}\right)} x_{n+i}\right)$ whenever $j, i \in \overline{1, n}$ with $j \neq i,\left[\mathrm{~T}_{\mathrm{K}}\left(x^{\left(k_{i} \varepsilon_{i}\right)} x_{2 n+1}\right), \mathrm{T}_{\mathrm{K}}\left(x_{n+i}\right)\right]=\mathrm{T}_{\mathrm{K}}\left(x^{\left(k_{i}-1\right) \varepsilon_{i}} x_{2 n+1}+x^{\left(k_{i} \varepsilon_{i}\right)} x_{n+i}\right)$ for $i \in \overline{1, n},\left[\mathrm{~T}_{\mathrm{K}}\left(x_{k} x_{l} x_{2 n+1}\right), \mathrm{T}_{\mathrm{K}}\left(x_{q}\right)\right]=\mathrm{T}_{\mathrm{K}}\left(x_{k} x_{l} x_{q}\right)$ for $k, l, q \in \overline{n+1,2 n}$, all the elements in $A$ may be generated by $\mathcal{M} \cup \mathcal{N} \cup \mathcal{P}$. Therefore,

$$
\mathrm{T}_{\mathrm{K}}\left(x^{(\delta)} x^{u}\right) \in \mathcal{L} \quad \text { for all } \delta \in \mathbb{A}(n, \underline{t}) \text { and all } u \in \mathbb{B}(n) \text { with }|u| \text { odd. }
$$

One can easily verify that $\mathrm{T}_{\mathrm{K}}\left(x^{(\delta)} x_{2 n+1}\right) \in \mathcal{L}$. Thus in order to complete the proof, it is sufficient to show that

$$
\mathrm{T}_{\mathrm{K}}\left(x^{(\delta)} x^{u} x_{n+j} x_{2 n+1}\right) \in \mathcal{L}
$$

for all $j \in \overline{1, n}, \delta \in \mathbb{A}(n, \underline{t})$ and $u \in \mathbb{B}(n)$ with $|u|$ odd.

For every $u \in \mathbb{B}(n)$, since $|u| \geq 1$, there exists $n+i \in u$. Then one computes

$$
\begin{aligned}
& {\left[\mathrm{T}_{\mathrm{K}}\left(x^{(\delta)} x^{u}\right), \mathrm{T}_{\mathrm{K}}\left(x_{n+i} x_{n+j} x_{2 n+1}\right)\right] } \\
= & \mathrm{T}_{\mathrm{K}}\left(\left(x^{\left(\delta-\varepsilon_{i}\right)} x^{u} x_{n+j} x_{2 n+1}-x^{\left(\delta-\varepsilon_{j}\right)} x^{u} x_{n+i} x_{2 n+1}\right.\right. \\
& \left.+\left(\sum_{i=1}^{n} \delta_{i}+|u|-2\right) x^{(\delta)} x^{u} x_{n+i} x_{n+j}\right)
\end{aligned}
$$




$$
=\mathrm{T}_{\mathrm{K}}\left(x^{\left(\delta-\varepsilon_{i}\right)} x^{u} x_{n+j} x_{2 n+1}\right) \text {. }
$$

It follows that $\mathrm{T}_{\mathrm{K}}\left(x^{\left(\delta-\varepsilon_{i}\right)} x^{u} x_{n+j} x_{2 n+1}\right) \in \mathcal{L}$. So it remains to show that

$$
\mathrm{T}_{\mathrm{K}}\left(x^{(\pi)} x^{u} x_{n+j} x_{2 n+1}\right) \in \mathcal{L} \quad \text { for all } j \in \overline{1, n} \text { and } u \in \mathbb{B}(n) \text { with }|u| \text { odd. }
$$

A routine computation shows that

$$
\begin{aligned}
& {\left[\mathrm{T}_{\mathrm{K}}\left(x^{\left(\pi-\varepsilon_{i}\right)} x_{2 n+1}\right), \mathrm{T}_{\mathrm{K}}\left(x^{\left(\varepsilon_{i}\right)} x^{u} x_{n+j} x_{2 n+1}\right)\right] } \\
= & (n+|u|+3) \mathrm{T}_{\mathrm{K}}\left(x^{(\pi)} x^{u} x_{n+j} x_{2 n+1}\right) .
\end{aligned}
$$

Here we have used the identity in the underlying field:

$$
\left(\sum_{k=1, k \neq i}^{n}\left(\begin{array}{c}
p^{t_{k}}-1 \\
1
\end{array}\right)+\left(\begin{array}{c}
p^{t_{i}}-2 \\
1
\end{array}\right)-|u|-2\right)\left(\begin{array}{c}
p^{t_{i}}-1 \\
1
\end{array}\right) \equiv n+|u|+3 \quad(\bmod p) .
$$

A similar computation gives

$$
\begin{aligned}
& {\left[\mathrm{T}_{\mathrm{K}}\left(x^{\left(\pi-2 \varepsilon_{i}\right)} x_{2 n+1}\right), \mathrm{T}_{\mathrm{K}}\left(x^{\left(2 \varepsilon_{i}\right)} x^{u} x_{n+j} x_{2 n+1}\right)\right] } \\
= & -(n+|u|+5) \mathrm{T}_{\mathrm{K}}\left(x^{(\pi)} x^{u} x_{n+j} x_{2 n+1}\right) .
\end{aligned}
$$

It follows from (2.1) and (2.2) that $\mathrm{T}_{\mathrm{K}}\left(x^{(\pi)} x^{u} x_{n+j} x_{2 n+1}\right) \in \mathcal{L}$ since $p>2$.

Summarizing, we have proved that $\mathrm{T}_{\mathrm{K}}\left(x^{(\delta)} x^{u}\right) \in \mathcal{L}$ for all $\delta \in \mathbb{A}(n, \underline{t}), u \in$ $\mathbb{B}^{1}$. Hence $\mathcal{L}=\mathfrak{K}$, completing the proof.

\subsection{Principal gradings and transitivity}

In order to compute the derivations, let us introduce a $\mathbb{Z}$-grading structure of $\mathfrak{K}$. Write $\xi:=|\pi|+n+1=\sum_{i=1}^{n} p^{t_{i}}+1$. In what follows, the symbol $\operatorname{deg}(x)$ always implies that $x$ is a $\mathbb{Z}$-homogeneous element with respect to the $\mathbb{Z}$-grading under consideration and $\operatorname{deg}(x)$ is the $\mathbb{Z}$-degree of $x$. By letting $\operatorname{deg}\left(x^{(\alpha)}\right):=|\alpha|$ for $\alpha \in \mathbb{A}$ and $\operatorname{deg}(u):=|u|+\delta_{2 n+1 \in u}$ for $u \in \mathbb{B}$, the divided power superalgebra has a $\mathbb{Z}$-grading structure, known as the principal grading,

$$
\mathcal{O}=\oplus_{i=0}^{\xi+1} \mathcal{O}_{i}, \quad \mathcal{O}_{i}=\operatorname{span}_{\mathbb{F}}\left\{x^{(\alpha)} x^{u} \mid \operatorname{deg}\left(x^{(\alpha)} x^{u}\right)=i, \alpha \in \mathbb{A}, u \in \mathbb{B}\right\} .
$$

Here, $\delta_{2 n+1 \in u}$ is, by definition, 1 if $2 n+1 \in u$ and 0 otherwise. In particular, $\operatorname{deg}\left(x_{i}\right)=1+\delta_{i, 2 n+1}$ for all $i \in \overline{1,2 n+1}$. By assigning $\operatorname{deg}\left(D_{i}\right)=-1-\delta_{i, 2 n+1}$ for $i \in \overline{1,2 n+1}$, the principal grading of $\mathcal{O}$ induces a $\mathbb{Z}$-grading structure on the generalized Witt superalgebra, known again as principal:

$W=\oplus_{i \geq-2} W_{i}, W_{i}:=\operatorname{span}_{\mathbb{F}}\left\{f D_{s} \mid f \in \mathcal{O}, s \in \overline{1,2 n+1}, \operatorname{deg}(f)+\operatorname{deg}\left(D_{s}\right)=i\right\}$.

It is routine to verify that the odd contact Lie superalgebra $K O$ is a $\mathbb{Z}$-graded subalgebra of $W$ with respect to the principal grading, that is,

$$
K O=\oplus_{i \geq-2} K O_{i}, \quad K O_{i}:=K O \cap W_{i} .
$$


Correspondingly, $\mathfrak{W}$ and $\mathfrak{K}$ have the principal grading structures:

$$
\begin{aligned}
& \mathfrak{W}=\oplus_{i \geq-1} \mathfrak{W}_{i}, \quad \mathfrak{W}_{i}:=\mathfrak{W} \cap W_{i}, \\
& \mathfrak{K}=\oplus_{i \geq-1} \mathfrak{K}_{i}, \quad \mathfrak{K}_{i}:=\mathfrak{K} \cap K O_{i} .
\end{aligned}
$$

In particular,

$$
\begin{aligned}
& \mathfrak{K}_{-1}=\operatorname{span}_{\mathbb{F}}\left\{\mathrm{T}_{\mathrm{K}}\left(x_{n+i}\right) \mid i \in \overline{1, n}\right\}, \\
& \mathfrak{K}_{0}=\operatorname{span}_{\mathbb{F}}\left\{\mathrm{T}_{\mathrm{K}}\left(x_{2 n+1}\right), \mathrm{T}_{\mathrm{K}}\left(x_{i} x_{n+j}\right) \mid i, j \in \overline{1, n}\right\} .
\end{aligned}
$$

We should note that $\mathrm{T}_{\mathrm{K}}$ is of degree -2 with respect to the principal gradings. For convenience, we list some formulas for later use $(i, j \in \overline{1, n})$ :

$$
\begin{aligned}
& \mathrm{T}_{\mathrm{K}}\left(x_{n+i}\right)=-D_{i}-x_{n+i} D_{2 n+1}, \\
& \mathrm{~T}_{\mathrm{K}}\left(x_{2 n+1}\right)=-\sum_{k=1}^{2 n} x_{k} D_{k}-2 x_{2 n+1} D_{2 n+1}, \\
& \mathrm{~T}_{\mathrm{K}}\left(x_{i} x_{n+j}\right)=\mathrm{T}_{\mathrm{H}}\left(x_{i} x_{n+j}\right)=x_{n+j} D_{n+i}-x_{i} D_{j} .
\end{aligned}
$$

Since both $\mathfrak{K}$ and $\mathfrak{W}$ are finite dimensional, viewing $\mathfrak{W}$ as a $\mathfrak{K}$-module by the adjoint representation, the derivation space $\operatorname{Der}(\mathfrak{K}, \mathfrak{W})$ inherits a $\mathbb{Z}$-grading from the principal gradings of $\mathfrak{K}$ and $\mathfrak{W}$ in the usual fashion:

$$
\operatorname{Der}(\mathfrak{K}, \mathfrak{W})=\oplus_{i \in \mathbb{Z}} \operatorname{Der}_{i}(\mathfrak{K}, \mathfrak{W}) .
$$

In particular, $\operatorname{Der}(\mathfrak{K})$ is a $\mathbb{Z}$-graded Lie algebra:

$$
\operatorname{Der}(\mathfrak{K})=\oplus_{i \in \mathbb{Z}} \operatorname{Der}_{i}(\mathfrak{K}) .
$$

Here we should mention that, to determine the derivations of $\mathfrak{K}$, we shall first determine the derivations from $\mathfrak{K}$ into $\mathfrak{W}$. This will largely simplify our computations since the elements in $\mathfrak{W}$ possess more concise expressions.

Write $\mathcal{G}$ for the centralizer of $\mathfrak{K}_{-1}$ in $\mathfrak{W}$, that is, $\mathcal{G}:=C_{\mathfrak{W}}\left(\mathfrak{K}_{-1}\right)$. By a lengthy but straightforward computation, we can verify the following:

Lemma 2.2. If $n$ is odd, then

$\mathcal{G}=\operatorname{span}_{\mathbb{F}}\left\{x^{u} D_{i}, x^{\omega} D_{i}, x^{v} D_{2 n+1} \mid i \in \overline{1, n}, 2 n+1 \notin u \in \mathbb{B}^{0} ; 2 n+1 \notin v \in \mathbb{B}^{1}\right\} ;$

If $n$ is even, then

$\mathcal{G}=\operatorname{span}_{\mathbb{F}}\left\{x^{u} D_{i}, x^{\omega} D_{2 n+1}, x^{v} D_{2 n+1} \mid i \in \overline{1, n}, 2 n+1 \notin u \in \mathbb{B}^{0} ; 2 n+1 \notin v \in \mathbb{B}^{1}\right\}$.

Similarly, one may easily give the centralizer of $\mathfrak{K}_{-1}$ in $\mathfrak{K}$ :

Lemma 2.3. If $n$ is odd, then

$$
C_{\mathfrak{K}}\left(\mathfrak{K}_{-1}\right)=\operatorname{span}_{\mathbb{F}}\left\{\mathrm{T}_{\mathrm{K}}\left(x^{u}\right) \mid 2 n+1 \notin u \in \mathbb{B}^{1}\right\} ;
$$

If $n$ is even, then

$$
C_{\mathfrak{K}}\left(\mathfrak{K}_{-1}\right)=\operatorname{span}_{\mathbb{F}}\left\{\mathrm{T}_{\mathrm{K}}\left(x^{\omega}\right), \mathrm{T}_{\mathrm{K}}\left(x^{u}\right) \mid 2 n+1 \notin u \in \mathbb{B}^{1}\right\} .
$$


Remark 2.4. We should mention that Lemma 2.3 implies that the principal grading of $\mathfrak{K}$ is not transitive and that $\mathfrak{K}$ is not simple.

Remark 2.5. It is a standard fact that $\mathcal{G}$ is a graded subalgebra of $\mathfrak{W}$ with respect to the principal gradings. By Lemma 2.3, $\mathcal{G}=\sum_{i} \mathcal{G}_{2 i+1}$, that is, $\mathcal{G}_{2 i}=0$ for all $i$. The same conclusion holds for $C_{\mathfrak{K}}\left(\mathfrak{K}_{-1}\right)$.

We close this section by introducing two technical lemmas. The first one is straightforward while the second is from [10, Proposition 2.1.6].

Lemma 2.6. Let $\phi \in \operatorname{Der}(\mathfrak{K}, \mathfrak{W})$ and $\phi\left(\mathfrak{K}_{-1}\right)=0$. If $E$ is an element of $\mathfrak{K}$ such that $\left[E, \mathfrak{K}_{-1}\right] \subset \operatorname{ker} \phi$, then $\phi(E) \in \mathcal{G}$.

Lemma 2.7. Any nonnegative $\mathbb{Z}$-homogeneous derivation from $\mathfrak{K}$ into $\mathfrak{W}$ modulo an inner derivation vanishes on $\mathfrak{K}_{-1}$.

\section{Proof of Theorems 1.1 and 1.2}

In this section, we completely determine the derivations from $\mathfrak{K}$ into $\mathfrak{W}$ (Theorem 1.1). We treat separately the derivations of nonnegative degree and of negative degree and obtain the following intermediate results:

Proposition 3.1. All the nonnegative homogeneous derivations from $\mathfrak{K}$ into $\mathfrak{W}$ are inner. That is,

$$
\operatorname{Der}^{\geq 0}(\mathfrak{K}, \mathfrak{W})=\operatorname{ad}\left(\mathfrak{W}^{\geq 0}\right) .
$$

Proposition 3.2. The negative homogeneous derivations from $\mathfrak{K}$ into $\mathfrak{W}$ are described as follows:

$$
\operatorname{Der}^{<0}(\mathfrak{K}, \mathfrak{W})=\operatorname{ad}_{-1} \oplus \operatorname{span}_{\mathbb{F}}\left\{\left(\operatorname{ad} D_{i}\right)^{p^{k_{i}}} \mid i \in \overline{1, n}, 1 \leq k_{i}<t_{i}\right\} .
$$

These two propositions will be proved in Subsections 3.1 and 3.2, respectively. Then we can give:

Proof of Theorem 1.1. This follows immediately from Propositions 3.1 and 3.2.

Every derivation of $\mathfrak{K}$ is a derivation from $\mathfrak{K}$ into $\mathfrak{W}$. Thereby Theorem 1.1 can be used to determine the derivation algebra of $\mathfrak{K}$.

Proof of Theorem 1.2. Let us show that

$$
\operatorname{Der}(\mathfrak{K})=\operatorname{ad}(\mathfrak{K}) \oplus \operatorname{span}_{\mathbb{F}}\left\{\left(\operatorname{ad} D_{i}\right)^{p^{k_{i}}} \mid i \in \overline{1, n}, 1 \leq k_{i}<t_{i}\right\} .
$$

First, consider the inclusion " $\subset$ ". Let $\phi$ be a homogeneous derivation. If $\phi$ is of degree $t \geq-1$, then by Theorem 1.1, there is an $E \in \mathfrak{W}$ such that $\phi=\operatorname{ad} E$. Note that $\mathfrak{K}$ is self-normalizing in $\mathfrak{W}$, that is, $\operatorname{Nor}_{\mathfrak{W}}(\mathfrak{K})=\mathfrak{K}$. One sees $E \in \mathfrak{K}$ and $\phi \in \operatorname{ad}(\mathfrak{K})$. If $\phi$ is of degree $t<-1$, then by Theorem 1.1, $\phi \in \operatorname{span}_{\mathbb{F}}\left\{\left(\operatorname{ad} D_{i}\right)^{p^{k_{i}}} \mid i \in \overline{1, n}, 1 \leq k_{i}<t_{i}\right\}$. Hence " $\subset$ " holds. The converse inclusion is immediate since $\left(\operatorname{ad} D_{i}\right)^{p^{k_{i}}}=\left(\operatorname{adT}_{\mathrm{K}}\left(x_{n+i}\right)\right)^{p^{k_{i}}}$ for any positive integer $k_{i}$. The dimensional formula for the outer derivation algebra is obvious and the proof is complete. 


\subsection{Derivations of nonnegative degree}

For convenience, write for $i \in \overline{1, n}$,

$$
\begin{aligned}
& \Delta_{i}:=\mathrm{T}_{\mathrm{K}}\left(x_{i} x_{i^{\prime}}\right)=x_{i^{\prime}} D_{i^{\prime}}-x_{i} D_{i}, \\
& \Delta_{2 n+1}:=\mathrm{T}_{\mathrm{K}}\left(x_{2 n+1}\right)=-\sum_{k=1}^{2 n} x_{k} D_{k}-2 x_{2 n+1} D_{2 n+1} .
\end{aligned}
$$

Note that

$$
\mathcal{T}:=\operatorname{span}_{\mathbb{F}}\left\{\Delta_{i}, \Delta_{2 n+1} \mid i \in \overline{1, n}\right\}
$$

is a maximal torus of dimension $n+1$ of $\mathfrak{K}_{0}$, known as standard. We also point out that $-\operatorname{ad} \Delta_{2 n+1}$ is the degree derivation of $\mathfrak{K}$ with respect to the principal grading, that is,

$$
\left[-\Delta_{2 n+1}, E\right]=k E \quad \text { for all } E \in \mathfrak{K}_{k} .
$$

In particular, the degree derivation is inner for this Lie algebra. We state a formula for later use: for $i \in \overline{1, n}$,

$$
\left[\mathrm{T}_{\mathrm{K}}\left(x^{(\alpha)} x^{u}\right), \Delta_{i}\right]=\left(\alpha_{i}-\delta_{n+i \in u}\right) \mathrm{T}_{\mathrm{K}}\left(x^{(\alpha)} x^{u}\right) .
$$

In order to prove Proposition 3.1, we first give a result pertaining to derivation vanishing:

Lemma 3.3. A nonnegative homogeneous derivation from $\mathfrak{K}$ into $\mathfrak{W}$ vanishes if and only if it vanishes on the non-positive part of $\mathfrak{K}$.

Proof. Suppose $\phi \in \operatorname{Der}_{t}(\mathfrak{K}, \mathfrak{W})$ with $t \geq 0$ and $\phi\left(\mathfrak{K}_{-1} \oplus \mathfrak{K}_{0}\right)=0$. In view of Theorem 2.1, we have to show that $\phi(\mathcal{N})=0=\phi(\mathcal{P})$.

Case 1. We first check that $\phi(\mathcal{N})=0$ or equivalently,

$$
\phi\left(\mathrm{T}_{\mathrm{K}}\left(x^{\left(a \varepsilon_{i}\right)} x_{2 n+1}\right)\right)=0 \quad \text { for all } i \in \overline{1, n}, 0 \leq a \leq \pi_{i} .
$$

For $a=1,2$, as in [8, Lemma 3.1], it is easy to see that (3.2) holds. Now we proceed by induction on $a>2$. Note that by induction hypothesis,

$$
\left[\phi\left(\mathrm{T}_{\mathrm{K}}\left(x^{\left(a \varepsilon_{i}\right)} x_{2 n+1}\right)\right), \mathrm{T}_{\mathrm{K}}\left(x_{n+j}\right)\right]=0 \quad \text { for all } j \in \overline{1, n} \text {. }
$$

By Lemma 2.6, it is sufficient to treat the following Subcases 1.1 and 1.2.

Subcase 1.1. Suppose

$$
\phi\left(\mathrm{T}_{\mathrm{K}}\left(x^{\left(a \varepsilon_{i}\right)} x_{2 n+1}\right)\right)=\sum_{r \in \overline{1, n}, u \in \mathbb{B}(n+1)} c_{u r} x^{u} D_{r}, \quad \text { where } c_{u r} \in \mathbb{F} .
$$

Applying $\phi$ to the equation that $\left[\mathrm{T}_{\mathrm{K}}\left(x^{\left(a \varepsilon_{i}\right)} x_{2 n+1}\right), \Delta_{i}\right]=a \mathrm{~T}_{\mathrm{K}}\left(x^{\left(a \varepsilon_{i}\right)} x_{2 n+1}\right)$, one gets

$$
\left[\sum_{r \in \overline{1, n}, u \in \mathbb{B}} c_{u r} x^{u} D_{r}, x_{i^{\prime}} D_{i^{\prime}}-x_{i} D_{i}\right]=a \sum_{r \in \overline{1, n}, u \in \mathbb{B}} c_{u r} x^{u} D_{r}
$$


and therefore,

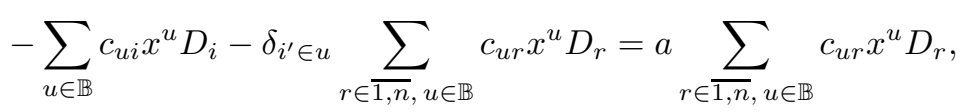

where $\delta_{i^{\prime} \in u}$ means that it is 1 if $i^{\prime} \in u$ and 0 otherwise. Comparing the coefficients, we have

$$
\begin{aligned}
& \left(a+\delta_{i^{\prime} \in u}\right) c_{u r}=0 \quad \text { for } r \in \overline{1, n} \backslash\{i\}, u \in \mathbb{B} \\
& \left(a+1+\delta_{i^{\prime} \in u}\right) c_{u i}=0 \quad \text { for } u \in \mathbb{B} .
\end{aligned}
$$

We distinguish two cases: (i) $a \equiv 0(\bmod p)$, and (ii) $a \not \equiv 0(\bmod p)$.

(i) Suppose $a \equiv 0(\bmod p)$. By $(3.4), c_{u i}=0$. Then

$$
\phi\left(\mathrm{T}_{\mathrm{K}}\left(x^{\left(a \varepsilon_{i}\right)} x_{2 n+1}\right)\right)=\sum_{\substack{i^{\prime} \in u \in \mathbb{B} \\ r \in \overline{1}, n}\{i\}} c_{u r} x^{u} D_{r}+\sum_{\substack{i^{\prime} \notin v \in \mathbb{B} \\ r \in \overline{1}, n \backslash\{i\}}} c_{v r} x^{v} D_{r} .
$$

By (3.3), one gets

$$
\phi\left(\mathrm{T}_{\mathrm{K}}\left(x^{\left(a \varepsilon_{i}\right)} x_{2 n+1}\right)\right)=\sum_{\substack{i^{\prime} \notin v \in \mathbb{B} \\ r \in 1, n}\{i\}} c_{v r} x^{v} D_{r} .
$$

For any fixed $v$ satisfying that $i^{\prime} \notin v$, since $|v|>3$, there exists $q \in v, q \neq 2 n+1$ and $q \neq i, i^{\prime}$ such that $\left[\mathrm{T}_{\mathrm{K}}\left(x^{\left(a \varepsilon_{i}\right)} x_{2 n+1}\right), \Delta_{q^{\prime}}\right]=0$. Applying $\phi$ to the above equation, we get $c_{v r}=0$ for $r \in \overline{1, n} \backslash\{i\}$. This proves (3.2).

(ii) Suppose $a \not \equiv 0(\bmod p)$. Similar to (i), from (3.3) and (3.4) one can show that (3.2) holds.

Subcase 1.2. Suppose

$$
\phi\left(\mathrm{T}_{\mathrm{K}}\left(x^{\left(a \varepsilon_{i}\right)} x_{2 n+1}\right)\right)=\sum_{u \in \mathbb{B}^{1}} c_{u 2 n+1} x^{u} D_{2 n+1}, \text { where } c_{u 2 n+1} \in \mathbb{F} .
$$

Similar to Subcase 1.1, it is easy to show (3.2).

Case 2. Let us show that $\phi(\mathcal{P})=0$, that is,

$$
\phi\left(\mathrm{T}_{\mathrm{K}}\left(x_{n+j} x_{n+k} x_{2 n+1}\right)\right)=0 \quad \text { for } j, k \in \overline{1, n} \text { with } j \neq k .
$$

We treat separately the two subcases of $t$ being even and $t$ being odd.

Subcase 2.1. Suppose $t$ is even. By Lemma 2.6, $\phi\left(\mathrm{T}_{\mathrm{K}}\left(x_{n+j} x_{n+k} x_{2 n+1}\right)\right)$ is of exactly one of the forms

$$
\sum_{\substack{r \in \overline{1, n} \\ 2 n+1 \in \omega \in \mathbb{B}^{0}}} c_{\omega r} x^{\omega} D_{r} \quad \text { and } \quad c_{\omega 2 n+1} x^{\omega} D_{2 n+1} .
$$

We only treat the first form since the other case can be treated similarly. Applying $\phi$ to the equation

$$
\left[\mathrm{T}_{\mathrm{K}}\left(x_{n+j} x_{n+k} x_{2 n+1}\right), \Delta_{j}\right]=-\mathrm{T}_{\mathrm{K}}\left(x_{n+j} x_{n+k} x_{2 n+1}\right),
$$


we obtain that $c_{\omega j}=0$. Consequently,

$$
\phi\left(\mathrm{T}_{\mathrm{K}}\left(x_{n+j} x_{n+k} x_{2 n+1}\right)\right)=\sum_{\substack{2 n+1 \in \omega \in \mathbb{B}^{0} \\ r \in \overline{1}, n \backslash\{j\}}} c_{\omega r} x^{\omega} D_{r} .
$$

By $|\omega| \geq 3,|\omega| \geq 5$, there exists $s \in \omega, s \neq 2 n+1$ and $s \neq n+j, n+k$ such that $\left[\mathrm{T}_{\mathrm{K}}\left(x_{n+j} x_{n+k} x_{2 n+1}\right), \Delta_{s^{\prime}}\right]=0$. Applying $\phi$ to the above equation, we have $c_{\omega r}=0$ for $r \in \overline{1, n} \backslash\{j\}$. Then (3.5) holds.

Subcase 2.2. Suppose $t$ is odd. By Lemma 2.6, $\phi\left(\mathrm{T}_{\mathrm{K}}\left(x_{n+j} x_{n+k} x_{2 n+1}\right)\right)$ is of exactly one of the forms

$$
\sum_{2 n+1 \notin u \in \mathbb{B}^{1}} c_{u 2 n+1} x^{u} D_{2 n+1} \text { and } \sum_{\substack{r \in \overline{1, n} \\ 2 n+1 \notin u \in \mathbb{B}^{0}}} c_{u r} x^{u} D_{r} .
$$

We only treat the first form since the other is similar. For any fixed $u$, by $|u| \geq 5$, there exists $q \in u, q \neq 2 n+1$ and $q \neq n+j, n+k$ such that $\left[\mathrm{T}_{\mathrm{K}}\left(x_{n+j} x_{n+k} x_{2 n+1}\right), \Delta_{q^{\prime}}\right]=0$. Applying $\phi$, one gets

$$
\left[\sum_{2 n+1 \notin u \in \mathbb{B}^{1}} c_{u 2 n+1} x^{u} D_{2 n+1}, x_{q} D_{q}-x_{q^{\prime}} D_{q^{\prime}}\right]=0 .
$$

Consequently, $-\sum_{2 n+1 \notin u \in \mathbb{B}^{1}} c_{u 2 n+1} x^{u} D_{2 n+1}=0$ and so $c_{u 2 n+1}=0$. This proves $\phi\left(\mathrm{T}_{\mathrm{K}}\left(x_{n+j} x_{n+k} x_{2 n+1}\right)\right)=0$.

We need, in addition, a reduction lemma of which the proof is completely analogous to [8, Lemmas 3.6 and 3.7]:

Lemma 3.4. A nonnegative derivation from $\mathfrak{K}$ into $\mathfrak{W}$ vanishing on $\mathfrak{K}_{-1}$ modulo an inner derivation vanishes on the non-positive part $\mathfrak{K}_{-1} \oplus \mathfrak{K}_{0}$ of $\mathfrak{K}$.

Proof of Proposition 3.1. The inclusion $\operatorname{adW}_{t} \subset \operatorname{Der}_{t}(\mathfrak{K}, \mathfrak{W})$ is immediate. On the other hand, for $\phi \in \operatorname{Der}_{t}(\mathfrak{K}, \mathfrak{W})$, by Lemma 2.7, there exists $E \in \mathfrak{W}_{t}$ such that $(\phi-\operatorname{ad} E)\left(\mathfrak{K}_{-1}\right)=0$. By Lemma 3.4 , there exists $D \in \mathcal{G}_{t}$ such that

$$
(\phi-\operatorname{ad} E-\operatorname{ad} D)\left(\mathfrak{K}_{-1} \oplus \mathfrak{K}_{0}\right)=0 .
$$

Then Lemma 3.3 and Theorem 2.1 ensure that $\phi-\operatorname{ad} E-\operatorname{ad} D=0$. Consequently, $\phi=\operatorname{ad} E+\operatorname{ad} D \in \operatorname{ad} \mathfrak{W}_{t}$ and the proof is complete.

\subsection{Derivations of negative degree}

In this subsection, we determine the homogeneous derivations of degree -1 , $-p^{d}$ for any positive integer $d$ and of the other negative degrees, respectively.

3.2.1. Degree $\mathbf{- 1}$. We first give a reduction lemma for degree -1 derivations.

Lemma 3.5. A derivation from $\mathfrak{K}$ into $\mathfrak{W}$ of degree -1 vanishes if and only if it vanishes on $\mathfrak{K}_{0}$. 
Proof. The "only if" direction is obvious. For the "if" direction, in view of Theorem 2.1, it is enough to show that $\phi(\mathcal{P})=0=\phi(\mathcal{N})$ for all $\phi \in \operatorname{Der}_{-1}(\mathfrak{K}, \mathfrak{W})$. Similar to $[8$, Lemma 4.2], it is easy to show that $\phi(\mathcal{N})=0$. Now let us show that $\phi(\mathcal{P})=0$, that is,

$$
\phi\left(\mathrm{T}_{\mathrm{K}}\left(x_{n+j} x_{n+k} x_{2 n+1}\right)\right)=0 \quad \text { for all } j, k \in \overline{1, n} \text { with } j \neq k .
$$

Since $\phi$ is of degree -1 , by Lemma $2.6, \phi\left(\mathrm{T}_{\mathrm{K}}\left(x_{n+j} x_{n+k} x_{2 n+1}\right)\right)$ is of exactly one of the forms

$$
\sum_{\substack{r \in \overline{1, n} \\ 2 n+1 \notin u \in \mathbb{B}^{0}}} c_{u r} x^{u} D_{r} \quad \text { and } \sum_{2 n+1 \notin u \in \mathbb{B}^{1}} c_{u 2 n+1} x^{u} D_{2 n+1} .
$$

We only treat the first form since the other is similar. Applying $\phi$ to the equation

$$
\left[\mathrm{T}_{\mathrm{K}}\left(x_{n+j} x_{n+k} x_{2 n+1}\right), \Delta_{j}\right]=-\mathrm{T}_{\mathrm{K}}\left(x_{n+j} x_{n+k} x_{2 n+1}\right),
$$

we obtain that for any given $u, c_{u r}=0$ for $r \in \overline{1, n} \backslash\{j\}$ if $n+j \notin u$ and $c_{u j}=0$ if $n+j \in u$.

Case 1. If $n+j \notin u$, then

$$
\phi\left(\mathrm{T}_{\mathrm{K}}\left(x_{n+j} x_{n+k} x_{2 n+1}\right)\right)=\sum_{2 n+1, n+j \notin u \in \mathbb{B}^{0}} c_{u j} x^{u} D_{j} .
$$

Since $|u|=2$, there exists $r \in u, r \neq n+j$ and $r \neq n+k$ such that

$$
\left[\mathrm{T}_{\mathrm{K}}\left(x_{n+j} x_{n+k} x_{2 n+1}\right), \Delta_{r^{\prime}}\right]=0 .
$$

Applying $\phi$, one gets $c_{u j}=0$. This proves (3.6).

Case 2. If $n+j \in u$, then

$$
\phi\left(\mathrm{T}_{\mathrm{K}}\left(x_{n+j} x_{n+k} x_{2 n+1}\right)\right)=\sum_{2 n+1 \notin u \in \mathbb{B}^{0}, r \in \overline{1, n} \backslash\{j\}} c_{u r} x^{u} D_{r} .
$$

Noting that

$$
\left[\mathrm{T}_{\mathrm{K}}\left(x_{n+j} x_{n+k} x_{2 n+1}\right), \Delta_{k}\right]=-\mathrm{T}_{\mathrm{K}}\left(x_{n+j} x_{n+k} x_{2 n+1}\right),
$$

one gets, for any given $u, c_{u r}=0$ for $r \in \overline{1, n} \backslash\{k\}$ if $n+k \notin u$. Thus

$$
\phi\left(\mathrm{T}_{\mathrm{K}}\left(x_{n+j} x_{n+k} x_{2 n+1}\right)\right)=\sum_{2 n+1 \notin u \in \mathbb{B}^{0}} c_{u k} x^{u} D_{k} .
$$

Since $n+k \notin u$ and $|u|=2$, there exists $q \in u, q \neq n+j$ and $q \neq n+k$ such that $\left[\mathrm{T}_{\mathrm{K}}\left(x_{n+j} x_{n+k} x_{2 n+1}\right), \Delta_{q^{\prime}}\right]=0$. Applying $\phi$ to the equation, one gets $c_{u k}=0$ and (3.6) holds. If $n+k \in u$, then $c_{u k}=0$. Next we consider only the case when $n+j \in u$ and $n+k \in u$. Suppose

$$
\phi\left(\mathrm{T}_{\mathrm{K}}\left(x_{n+j} x_{n+k} x_{2 n+1}\right)\right)=\sum_{\substack{n+j, n+k \in u \in \mathbb{B}^{0} \\ r \in \overline{1}, n \backslash\{j, k\}}} c_{u r} x^{u} D_{r} .
$$


For any fixed $u$ satisfying $n+j \in u$ and $n+k \in u$, choose any $t \in \overline{1, n} \backslash\{j, k\}$. Applying $\phi$ to the equation $\left[\mathrm{T}_{\mathrm{K}}\left(x_{n+j} x_{n+k} x_{2 n+1}\right), \Delta_{t}\right]=0$, we can obtain that $c_{u t}=0$ for $t \in \overline{1, n} \backslash\{j, k\}$. This proves (3.6).

Proposition 3.6. Any derivation from $\mathfrak{K}$ into $\mathfrak{W}$ of degree -1 is inner.

Proof. For $\phi \in \operatorname{Der}_{-1}(\mathfrak{K}, \mathfrak{W})$, we may suppose

$$
\phi\left(\mathrm{T}_{\mathrm{K}}\left(x_{2 n+1}\right)\right)=\sum_{r \in \overline{1, n}} c_{r} D_{r}+\sum_{s \in \overline{n+1,2 n}} d_{s} x_{s} D_{2 n+1}
$$

where $c_{r}, d_{s} \in \mathbb{F}$. Let $E:=-\left(\sum_{r \in \overline{1, n}} c_{r} D_{r}+\sum_{s \in \overline{n+1,2 n}} d_{s} x_{s} D_{2 n+1}\right)$. Writing $\psi:=\phi-\operatorname{ad} E$, one sees $\psi\left(\mathrm{T}_{\mathrm{K}}\left(x_{2 n+1}\right)\right)=0$. Then we have

$$
\left[\psi\left(\mathrm{T}_{\mathrm{K}}\left(x_{i} x_{j}\right)\right), \mathrm{T}_{\mathrm{K}}\left(x_{2 n+1}\right)\right]=0 \quad \text { for } i \in \overline{1, n}, j \in \overline{n+1,2 n} \text {. }
$$

On the other hand, a simple computation shows that

$$
\left[\psi\left(\mathrm{T}_{\mathrm{K}}\left(x_{i} x_{j}\right)\right), \mathrm{T}_{\mathrm{K}}\left(x_{2 n+1}\right)\right]=-\psi\left(\mathrm{T}_{\mathrm{K}}\left(x_{i} x_{j}\right)\right) \quad \text { for } i \in \overline{1, n}, j \in \overline{n+1,2 n} \text {. }
$$

Therefore, $\psi\left(\mathrm{T}_{\mathrm{K}}\left(x_{i} x_{j}\right)\right)=0$ for $i \in \overline{1, n}, j \in \overline{n+1,2 n}$. This implies that $\psi\left(\mathfrak{K}_{0}\right)=0$. By Lemma 3.5, we get $\psi=0$. Consequently, $\phi=\operatorname{ad} E \in \operatorname{ad}_{-1}$. The proof is complete.

Next, we compute the derivations of degree less than -1 .

3.2.2. Degree $-\boldsymbol{p}^{\boldsymbol{d}}$. We need a reduction lemma for derivations of degree less than -1 .

Lemma 3.7. A homogenous derivation from $\mathfrak{K}$ into $\mathfrak{W}$ having degree $t<-1$ vanishes if and only if it vanishes on the elements of form $\mathrm{T}_{\mathrm{K}}\left(x^{\left(t \varepsilon_{i}\right)} x_{2 n+1}\right)$ with $i \in \overline{1, n}$.

Proof. The "only if" direction is obvious. Conversely, let $\phi$ be a derivation vanishing on the elements indicated above. By Theorem 2.1, it suffices to show that $\phi(\mathcal{P})=0=\phi(\mathcal{N})$. But this can be proved completely analogously to $[8$, Lemma 4.5].

Proposition 3.8. Let $t=p^{d}$, where $d$ is a positive integer. Then

$$
\operatorname{Der}_{-t}(\mathfrak{K})=\operatorname{span}_{\mathbb{F}}\left\{\left(\operatorname{ad} D_{i}\right)^{t} \mid i \in \overline{1, n}\right\} \text {. }
$$

Proof. Let $\phi \in \operatorname{Der}_{-t}(\mathfrak{K})$. Given any $i \in \overline{1, n}$, one may suppose

$$
\phi\left(\mathrm{T}_{\mathrm{K}}\left(x^{\left(t \varepsilon_{i}\right)} x_{n+i}\right)\right)=\sum_{j \in \overline{1, n}} c_{j} D_{j}+\sum_{j^{\prime} \in \overline{n+1,2 n}} c_{j} x_{j^{\prime}} D_{2 n+1}, \quad \text { where } c_{j} \in \mathbb{F} \text {. }
$$

Since $t \equiv 0(\bmod p)$, we have

$$
\left[\mathrm{T}_{\mathrm{K}}\left(x^{\left(t \varepsilon_{i}\right)} x_{n+i}\right), \Delta_{i}\right]=-\mathrm{T}_{\mathrm{K}}\left(x^{\left(t \varepsilon_{i}\right)} x_{n+i}\right) .
$$

Applying $\phi$ to the equation above, one gets

$$
\phi\left(\mathrm{T}_{\mathrm{K}}\left(x^{\left(t \varepsilon_{i}\right)} x_{n+i}\right)\right)=c_{i} D_{i}+c_{i} x_{n+i} D_{2 n+1} .
$$


To finish, we produce a new derivation $\psi:=\phi+\sum_{r \in \overline{1, n}} c_{r}\left(\operatorname{ad} D_{r}\right)^{t}$. One may verify that

$$
\psi\left(\mathrm{T}_{\mathrm{K}}\left(x^{\left(t \varepsilon_{i}\right)} x_{n+i}\right)\right)=0 \text { for all } i \in \overline{1, n} .
$$

Fixing any $i \in \overline{1, n}$ and picking any $j \in \overline{1, n} \backslash\{i\}$, one gets

$$
\mathrm{T}_{\mathrm{K}}\left(x^{\left(t \varepsilon_{i}\right)} x_{n+j}\right)=\left[\mathrm{T}_{\mathrm{K}}\left(x_{i} x_{n+j}\right), \mathrm{T}_{\mathrm{K}}\left(x^{\left(t \varepsilon_{i}\right)} x_{n+i}\right)\right] .
$$

From (3.7) and (3.8), one may deduce that

$$
\psi\left(\mathrm{T}_{\mathrm{K}}\left(x^{\left(t \varepsilon_{i}\right)} x_{n+j}\right)\right)=0 \quad \text { for all } i, j \in \overline{1, n} \text {. }
$$

Note that for any $i, j \in \overline{1, n}$ and any positive integer $k$,

$$
\left[\mathrm{T}_{\mathrm{K}}\left(x^{\left(k \varepsilon_{i}\right)} x_{2 n+1}\right), \mathrm{T}_{\mathrm{K}}\left(x_{n+j}\right)\right]=\delta_{i, j} \mathrm{~T}_{\mathrm{K}}\left(x^{(k-1) \varepsilon_{i}} x_{2 n+1}\right)+\mathrm{T}_{\mathrm{K}}\left(x^{\left(k \varepsilon_{i}\right)} x_{n+j}\right) \text {. }
$$

From (3.9) and (3.10), one may show that

$$
\left[\psi\left(\mathrm{T}_{\mathrm{K}}\left(x^{\left(t \varepsilon_{i}\right)} x_{2 n+1}\right)\right), \mathrm{T}_{\mathrm{K}}\left(x_{n+j}\right)\right]=0 \quad \text { for all } i, j \in \overline{1, n},
$$

that is, $\psi\left(\mathrm{T}_{\mathrm{K}}\left(x^{\left(t \varepsilon_{i}\right)} x_{2 n+1}\right)\right) \in \mathcal{G}$. Consequently,

$$
\psi\left(\mathrm{T}_{\mathrm{K}}\left(x^{\left(t \varepsilon_{i}\right)} x_{2 n+1}\right)\right) \in \mathcal{G} \cap \mathfrak{W}_{0}=\mathcal{G}_{0} \quad \text { for all } i \in \overline{1, n} .
$$

By Lemma $2.5, \mathcal{G}_{0}=0$ and thus $\psi(\mathcal{N})=0$. By Lemma 3.7, $\psi=0$ and therefore,

$$
\phi=-\sum_{r \in \overline{1, n}} c_{r}\left(\operatorname{ad} D_{r}\right)^{t} \in \operatorname{span}_{\mathbb{F}}\left\{\left(\operatorname{ad} D_{i}\right)^{t} \mid i \in \overline{1, n}\right\}
$$

The converse is obvious since a $p$-power of a derivation is again a derivation.

3.2.3. Other negative degrees. In this subsection, we prove that the derivations from $\mathfrak{K}$ into $\mathfrak{W}$ having degree $-t$ are trivial, where $t>1$ is not a p-power.

Proposition 3.9. $\operatorname{Der}_{-t}(\mathfrak{K}, \mathfrak{W})=0$, where $t>1$ is not a $p$-power.

Proof. Let $\phi \in \operatorname{Der}_{-t}(\mathfrak{K}, \mathfrak{W})$. We distinguish two cases:

Case 1. Suppose $t \not \equiv 0(\bmod p)$. Consider the degree derivation (3.1). We have for any $k$,

$$
(k-t) \phi(E)=\left[\phi(E), \Delta_{2 n+1}\right]=k \phi(E) \quad \text { for all } E \in \mathfrak{K}_{k} .
$$

Consequently, $\phi=0$.

Case 2. Suppose $t \equiv 0(\bmod p)$. We want to check that $\phi$ fulfills the conditions of Lemma 3.7, that is,

$$
\phi\left(\mathrm{T}_{\mathrm{K}}\left(x^{\left(t \varepsilon_{i}\right)} x_{2 n+1}\right)\right)=0 \quad \text { for all } i \in \overline{1, n} .
$$


Consider the $p$-adic form $t=\sum_{s=1}^{r} a_{s} p^{s}$, where $0 \leq a_{s}<p$ and $a_{r} \neq 0$. Observing the $\mathbb{Z}$-degrees, one gets

$$
\phi\left(\mathrm{T}_{\mathrm{K}}\left(x^{\left(\left(t-p^{r}+1\right) \varepsilon_{i}\right)} x_{2 n+1}\right)\right)=0=\phi\left(\mathrm{T}_{\mathrm{K}}\left(x^{\left(p^{r} \varepsilon_{i}\right)} x_{i^{\prime}}\right)\right) .
$$

Note that $\left(\begin{array}{c}t \\ p^{r}\end{array}\right) \not \equiv 0(\bmod p)$ and

$$
\begin{aligned}
& {\left[\mathrm{T}_{\mathrm{K}}\left(x^{\left(\left(t-p^{r}+1\right) \varepsilon_{i}\right)} x_{2 n+1}\right), \mathrm{T}_{\mathrm{K}}\left(x^{\left(p^{r} \varepsilon_{i}\right)} x_{i^{\prime}}\right)\right] } \\
= & \left(\begin{array}{c}
t \\
p^{r}
\end{array}\right) \mathrm{T}_{\mathrm{K}}\left(x^{\left(t \varepsilon_{i}\right)} x_{2 n+1}\right)+\left(\begin{array}{c}
t+1 \\
p^{r}
\end{array}\right) \mathrm{T}_{\mathrm{K}}\left(x^{\left((t+1) \varepsilon_{i}\right)} x_{i^{\prime}}\right) .
\end{aligned}
$$

Therefore, to show (3.12), it is sufficient to verify that

$$
\phi\left(\mathrm{T}_{\mathrm{K}}\left(x^{\left((t+1) \varepsilon_{i}\right)} x_{i^{\prime}}\right)\right)=0 .
$$

To do so, we first assert that $\phi\left(\mathrm{T}_{\mathrm{K}}\left(x^{\left(t \varepsilon_{i}\right)} x_{i^{\prime}}\right)\right)=0$. Indeed,

$$
\left[\mathrm{T}_{\mathrm{K}}\left(x^{\left(\left(t-p^{r}+1\right) \varepsilon_{i}\right)} x_{i^{\prime}}\right), \mathrm{T}_{\mathrm{K}}\left(x^{\left(p^{r} \varepsilon_{i}\right)} x_{i^{\prime}}\right)\right]=\left(\left(\begin{array}{c}
t \\
p^{r}
\end{array}\right)-\left(\begin{array}{c}
t \\
p^{r}-1
\end{array}\right)\right) \mathrm{T}_{\mathrm{K}}\left(x^{\left(t \varepsilon_{i}\right)} x_{i^{\prime}}\right) .
$$

The assertion follows since $\left(\begin{array}{c}t \\ p^{r}\end{array}\right) \not \equiv 0(\bmod p)$ and $\left(\begin{array}{c}t \\ p^{r}-1\end{array}\right) \equiv 0(\bmod p)$. Now, keeping in mind that $t \equiv 0(\bmod p)$, one computes

$$
\mathrm{T}_{\mathrm{K}}\left(x^{\left((t+1) \varepsilon_{i}\right)} x_{i^{\prime}}\right)=-\left[\mathrm{T}_{\mathrm{K}}\left(x^{\left(t \varepsilon_{i}\right)} x_{i^{\prime}}\right), \mathrm{T}_{\mathrm{K}}\left(x^{\left(2 \varepsilon_{i}\right)} x_{i^{\prime}}\right)\right] .
$$

Consequently, (3.13) holds. The proof is complete.

\section{References}

[1] S. Bouarroudj, P. Grozman, and D. Leites, Classification of finite dimensional modular Lie superalgebras with indecomposable Cartan matrix, Symmetry Integrability Geom. Methods Appl. 5 (2009), 63 pages.

[2] S. Bouarroudj and D. Leites, Simple Lie superalgebras and nonintegrable distributions in characteristic $p$, J. Math. Sci. 141 (2007), no. 4, 1390-1398.

[3] M. J. Celousov, Derivations of Lie algebras of Cartan type, Izv. Vyssh. Uchebn. Zaved. Mat. 98 (1970), 126-134.

[4] J.-Y. Fu, Q.-C. Zhang, and C.-P. Jiang, The Cartan-type modular Lie superalgebra KO, Comm. Algebra 34 (2006), no. 1, 107-128.

[5] V. G. Kac, Lie superalgebras, Adv. Math. 26 (1977), no. 1, 8-96.

[6] W.-D. Liu and B.-L. Guan, Derivations from the even parts into the odd parts for Lie superalgebras $W$ and $S$, J. Lie Theory 17 (2007), no. 3, 449-468.

[7] W.-D. Liu and Y.-H. He, Finite-dimensional special odd Hamiltonian superalgebras in prime characteristic, Commun. Contemp. Math. 11 (2009), no. 4, 523-546.

[8] W.-D. Liu, X.-Y. Hua, and Y.-C. Su, Derivations of the even part of the odd Hamiltonian superalgebra in modular case, Acta Math Sin. 25 (2009), no. 3, 355-378.

[9] W.-D. Liu and Y.-Z. Zhang, Outer derivation algebras of finite-dimensional Cartan-type modular Lie superalgebras, Comm. Algebra 33 (2005), no. 7, 2131-2214.

[10] Derivations of the even parts for modular Lie superalgebras of Cartan type $W$ and $S$, Internat. J. Algebra Comput. 17 (2007), no. 4, 661-714.

[11] W.-D. Liu, Y.-Z. Zhang, and X.-L. Wang, The derivation algebra of the Cartan-type Lie superalgebra HO, J. Algebra 273 (2004), no. 1, 176-205. 
[12] H. Strade, Simple Lie algebras over fields of positive characteristic. I, Structure Theory, Walter de Gruyter, Berlin and New York, 2004.

[13] H. Strade and R. Farnsteiner, Modular Lie Algebras and Their Representations, Monographs and Texbooks in Pure and Appl. Math. Vol. 116. Marcel Dekker Inc. 1988.

[14] W.-Q. Wang and L. Zhao, Representations of Lie superalgebras in prime characteristic I, arXiv: 0808.0046v[math.RT], 12 Jan 2009.

[15] C.-W. Zhang, Simple modules for the restricted Lie superalgebras sl(n, 1), J. Pure Appl. Algebra 213 (2009), no. 5, 756-765.

[16] Y.-Z. Zhang, Finite-dimensional Lie superalgebras of Cartan type over fields of prime characteristic, Chinese Sci. Bull. 42 (1997), no. 9, 720-724.

[17] $\_$, Graded modules for $\mathbb{Z}$-graded Lie superalgebras $W(n)$ and $S(n)$ of Cartan type, Kexue Tongbao (Chinese) 40 (1995), no. 20, 1829-1832.

[18] $\longrightarrow \mathbb{Z}$-graded module of Lie superalgebra $H(n)$ of Cartan type, Chinese Sci. Bull. 41 (1996), no. 10, 813-817.

YAN CAO

College of RongCheng

Harbin University of Science and Technology

Rongcheng 264300, P. R. China

E-mail address: caoyan_hrbust@qq.com

XiUmei Sun

School of Mathematical Sciences

HARbin NORMAL UNIVERSITY

Harbin 150025, P. R. China

E-mail address: sxm@hrbnu.edu.cn

JiXIA YUAN

School of Mathematical Sciences

HEILONGJIANG UNIVERSITY

Harbin 150080, P. R. China

E-mail address: $876961471 @ q q . c o m$ 